\title{
Umsetzen praktischer Energiebedarfsforschung in strategischen Energiemodellen
}

Dr.-Ing. A. Voß, Jülich, Dr. E. Geißler, Jülich

\section{Einleitung}

Im Vorwort zum Programm dieser Tagung heißt es: "Die Sicherstellung unserer künftigen Energieversorgung bedarf einer sorgfältigen Planung. Planung aber braucht Grundlagen, auf denen sie aufbauen, Zahlen, mit denen sie arbeiten kann. Modelle und Scenarien, wie sie als wichtige Planungs- und Entscheidungshilfen erstellt werden, scheitern immer wieder daran, daß letztlich vielfach praxisorientierte, zuverlässige, detaillierte Fakten über den Energiebedarf und seine technischen, wirtschaftlichen, sozialen und ökologischen Interdependenzen fehlen". Damit ist die Situation desjenigen, der im Bereich der Energiewirtschaft oder der Energiepolitik mit Planungsaufgaben betraut ist, weitgehend zutreffend, wenn auch etwas drastisch gekennzeichnet. Trotz einiger Fortschritte in den letzten Jahren ist unser Wissen über das, was man allgemein Energiebedarf nennt, sowie über seine individuellen, gesellschaftlichen und ökonomischen Bestimmungsgründe im Vergleich zu unserem Wissen über die Techniken der Umwandlung, des Transports und der Verteilung von Energie wohl eher bescheiden zu nennen und keinesfalls ausreichend für eine der komplexen Energieproblematik gerechtwerdende Planung. Vor diesem Hintergrund kann es auch nicht verwundern, daß in der öffentlichen Energiediskussion die Vorstellungen darüber, was wir an Energie heute und in Zukunft eigentlich brauchen, hochgradig kontrovers sind. Dabei wird die Diskussion noch durch eine unsachgerechte und unklare Verwendung der Begriffe erschwert, was wohl ein weiteres Indiz für unser mangelhaftes Verständnis gegenüber Energiebedarf und Energienachfrage ist. So z.B. sprechen wir von Energieverbrauch, obwohl Energie nach dem ersten Hauptsatz der Thermodynamik nicht verbraucht werden kann. Wir sprechen von Energiebedarf und meinen einmal Primärenergie, ein andermal Endenergie und sind uns dabei in der Regel nicht bewußt, daß wir ja eigentlich keinen $B e-$ darf an Energie, sondern an physiologisch günstig temperierten Räumen, Mobilitäts- und Kommunikationswünsche, sowie Bedarf an anderen Gütern und Dienstleistungen haben, deren Bereitstellung mit einem mehr oder weniger großen Umsatz an Energie verbunden ist.

Im folgenden wird zum einen auf die zuvor angesprochenen grundsätzlichen Probleme der Behandlung von "Energiebedarf" in strategischen Energiemodellen eingegangen und zum anderen werden die heute gebräuchlichen Verfahren der modellmä- 
Bigen Abbildung des "Energiebedarfs" diskutiert, um dann aus der Sicht desjenigen, der mit Fragen der Energieplanung befaßt ist, Anforderungen an die Energiebedarfsforschung abzuleiten. Zunächst aber seien einige Anmerkungen zum Sinn und Zweck, zu den Möglichkeiten und Grenzen strategischer Energiemodelle gemacht.

2. Strategische Energiemodelle: Ziele, Möglichkeiten und Grenzen

Energiemodelle oder ganz allgemein Modelle sind vereinfachte, auf das Wesentliche abstrahierte Abbilder komplexer realer Systeme [1]. Sie dienen der Untersuchung, dem besseren Verständnis für Struktur und Verhalten des jeweils abgebildeten realen Systems. Unter strategischen Energiemodellen sollen hier solche Modelle verstanden werden, die das Energiesystem oder wesentliche Teile des Energiesystems beschreiben und insbesondere in der Lage sind, mögliche Entwicklungen des Energiesystems und die daraus folgenden Konsequenzen quantitativ zu antizipieren. Ihr Einsatzbereich ist in der Energiewirtschaft oder Energiepolitik da anzusiedeln, wo strategische, d.h. oftmals weit in die Zukunft reichende Entscheidungen zu fällen sind, z.B. bei der Kraftwerksausbauplanung oder der Prioritätensetzung im Forschungsbereich.

Strategische Planungen hat es im Energiebereich schon immer gegeben. In der Vergangenheit aber war die Anwendung mathematischer Modelle vornehmlich auf die Erstellung von Prognosen, also auf die Vorhersage der zukünftigen Entwicklung, z.B. des Primärenergieverbrauchs oder des Verbrauchs einzelner Endenergieträger, ausgerichtet. Ein Vergleich der Energieprognosen der vergangenen drei Jahrzehnte [2] mit der tatsächlichen Entwicklung zeigt, daß die Prognostiker nicht sehr erfolgreich waren. Die wenigen Treffer, die zu verzeichnen waren, sind wohl mehr durch Zufall bedingt. Eine Analyse der Prognosen der zurückliegenden Jahrzehnte zeigt, daß nicht nur die aufgetretenen Krisen oder unerwarteten Ereignisse, wie z.B. die Ölkrise des Jahres 1973, nicht vorhergesagt werden konnten, sondern daß es selbst in Zeiten stetiger Entwicklungen nicht gelang, die Zukunft des Energiesystems auch über kurze Zeiträume hinweg vorherzusagen.

Ohne hier ausführlicher auf die allgemeine Problematik von Prognosen einzugehen, kann man aus der negativen Erfahrung der Vergangenheit das Fazit ziehen, daß in Anbetracht der vielfältigen Unsicherheiten die Vorhersage der Entwicklung des Energiesystems, z.B. die Festlegung der Kraftwerksleistung, die im Jahre 2000 installiert oder der Menge Öl, die im Jahre 2030 verbraucht werden wird, ein wenig nützliches Unterfangen ist. Die zutreffende Prognose, im eigentlichen Sinne des Wortes verstànden, ist aber auch nicht das Ziel einer Beschäftigung mit der Zukunft. Vielmehr, dies muß deutlich herausgestellt werden, beschäftigen wir uns mit der Zukunft, um Informationen und Erkenntnisse über mögliche Entwicklungen zu 
gewinnen, die notwendig und hilfreich bei der Festlegung der heute zu treffenden Entscheidungen sind, und zwar unter dem Gesichtspunkt, daß diese Entscheidungen zu einem wesentlich späteren Zeitpunkt nicht zu bereuen sein werden. Ubertragen auf den Energiebereich heißt dies: Wir beschäftigen uns mit der zukünftigen Entwicklung des Energiesystems, um strategisch relevante Einsichten zu gewinnen, ob wir, wie oben angesprochen, beispielsweise ein Kraftwerk zu bauen sollen oder ob heute Maßnahmen, und wenn ja, welche, zur Reduktion unserer Ölabhängigkeit eingeleitet werden müssen.

Im Unterschied zu Prognosen soll der Versuch, komplexe, in die Zukunft reichende Entwicklungen und Möglichkeiten hinsichtlich ihrer Beeinflußbarkeit zu analysieren, um Rückschlüsse auf heute zu treffende Entscheidungen zu ziehen, als "Systematische Zukunf tsanalyse" bezeichnet werden. Dabei wird weder die Vergangenheit fort-noch die Zukunft normativ festgeschrieben, sondern unter Berücksichtigung der Unsicherheit werden mögliche zukünftige Entwicklungen analysiert, um Handlungsnotwendigkeiten abzuleiten. Die Entwicklung und Anwendung strategischer Energiemodelle hat nur dann einen Sinn und rechtfertigt den damit verbundenen Aufwand, wenn sie auf dieses Ziel, d.h. auf die Erarbeitung rational begründbarer Entscheidungshilfen ausgerichtet ist.

Jede Strategiebetrachtung zur Auslegung des künftigen Energiesystems, ob sie sich auf die gesamte Energiewirtschaft, auf die gewichtigen Versorgungszweige und Abnehmerbereiche, auf die technischen Entwicklungslinien oder auf technisch-wirtschaftliche Implikationen erstreckt, sieht sich allerdings der zentralen Frage nach Höhe, Struktur und Modalitäten der Energiebedarfsentwicklung konfrontiert. War es in der Vergangenheit, angesichts einer stetigen wirtschaftlichen Entwicklung und einer kontinuierlichen Energiepreisentwicklung, noch möglich, mit relativ einfachen Ansätzen die Nachfrageentwicklung nach Energieträgern zu beschreiben die über lange Zeit brauchbare Annahme einer Verdopplungszeit der Elektrizitätsnachfrage von sieben Jahren sei hier als Beispiel angeführt - so haben uns die drastischen Veränderungen der energiewirtschaftlichen Situation in den letzten Jahren wohl erst vor Augen geführt, wie wenig wir eigentlich über den sog. "Energiebedarf" und seine Determinanten wissen. Es fehlt dabei nicht nur an statistisçhem Zahlenmaterial, sondern auch an ausreichenden Erkenntnissen über die funktionellen und die quantitativen Zusammenhänge zwischen dem sog. "Energiebedarf" und seinen Bestimmungsgrößen. Erwähnt seien hier auch die nur unzureichend bekannten Zusammenhänge der gegenseitigen Substitution von Energie, Kapital, Arbeit und Know-how oder der Zusammenhang zwischen Energieumsatz und Aufwand an Zeit, die eine der wenigen wirklichen limitierten Ressourcen des Menschen ist. 
3. Erfassung des Energiebedarfs in Energiemodellen

Bei der Erstellung und Anwendung von Energiemodellen kommt der sachlogisch richtigten, ausreichend detaillierten Erfassung und Abbildung des Komplexes "Energiebedarf" eine zentrale Rolle zu, weil damit ja sowohl die quantitativen wie auch qualitativen Anforderungen an das eigentliche Energieversorgungssystem abgesteckt werden. Die Bedarfsentwicklung erfolgt aber keineswegs autonom oder gemäß einfachen, leicht durchschau- und quantifızierbaren Mechanismen. Sie wird vielmehr von einer Vielzahl okonomischer, technischer aber auch sozialer Faktoren beeınflußt. Die problemadäquate Behandlung des Energiebedarfs ist dabei ebenfalls mit der jeweiligen strategischen Fragestellung zu verknüpfen. Es ist somıt sachangemessen und auch üblich, in Uodellen den Komplex Energiebedarf mit sehr unterschiedlichem Detaillierungsgrad abzubilden.

\section{Der makroökonomische Ansatz}

Dieser stellt die Leistungsfähigkeit der gesamten Volkswirtschaft in Abhängigkeit von der Wirtschaftsstruktur und vorn sektoralen Produktionsfaktoreinsatz, einschließlich Energieeinsatz, in den Vordergrund der Betrachtungen. Ökonomisch gesehen, ist die im Rahmen des Wirtschaftsgeschehens nachgefragte oder auch benötigte Energie ein Produktionsfaktor, der im Substitutionswettbewerb mit anderen Produktionsfaktoren, üblicherweise Arbeit und Kapital steht $[3,4]$. Sowohl durch die Verringerung der Energieintensität der einzelnen Produktionsprozesse über technisch-organisatorischen Maßnahmen als auch durch strukturelle Veränderungen des Produktionsspektrums zugunsten weniger energieintensiver Güter könnte im Prinzip ein höherer Produktionswert bei geringerem Energieverbrauch erzielt werden. Strukturverändernde Investitionen und die auf dem Wege der technischen Innovation zu erzielenden Nutzungsgradverbesserungen sind gemeint, wenn in diesem Zusammenhang von einer Substitution der Energie durch Kapital die Rede ist. Der makroökonomische Ansatz bedient sich sowohl zur Beschreibung des gesamten, ggf. sektoral gegliederten Produktionsausstoßes wie auch zur Beschreibung der preisabhängigen Substitution zwischen den Produktionsfaktoren Kapital, Arbeit und Energie sogenannter Produktionsfunktionen, also funktionaler Abhängigkeiten zwischen den Produktionseinsatz- und Ausstoßmengen, die im allgemeinen einheitlich monetär bewertet werden. In diesen Beziehungen können die intrasektoralen Lieferverflechtungen und somit sektoral unterschiedliche Energieintensitäten berücksichtigt werden. Die Beziehungen zum preisabhängigen Substitutions- und Investitionsverhalten der Wirtschaftssubjekte sind stark durch die volkswirtschaftliche Hypothesenbildung geprägt. Die Parameter der Produktionsfunktion werden in der Regel anhand statistischer Zeitreihen geschätzt. Es sei hier noch angemerkt, daß Produk- 
tionsfunktionen, die die Energie explizit als Produktionsfaktor enthalten, erst in den letzten Jahren Eingang in die ökonometische Modellentwicklung gefunden haben.

Der makroökonomische Ansatz der Darstellung des Energiebedarfs durch ökonometrische Produktionsfaktoren hat wie jeder Modellansatz hinsichtlich seiner Aussage- und Anwendungsmöglichkeit konzeptionsbedingte Grenzen. Sie resultieren vornehmlich aus dem hohen Aggregationsgrad, der auch dann noch gegeben ist, wenn die Produktion eines Wirtschaftssektors oder der gesamten Volkswirtschaft nicht, wie es häufig geschieht, mit nur einer Produktionsfunktion beschrieben wird. Diese geringe Differenzierung kann der unterschiedlichen thermodynamischen Qualität verschiedener Energieformen, z.B. der von Elektrizität und Niedertemperaturwärme, nicht in befriedigendem Maße Rechnung tragen. Des weiteren erfaßt der hochaggregierte Ansatz den Energieeinsatz nicht in seinen verschiedenen Verwendungszwecken und somit nicht die jeweils unterschiedlichen technischen und ökonomischen Möglichkeiten und Grenzen der Substitution von Energie, Arbeit und Kapital. Als problematisch erweist sich ferner, daß der Energiebedarf ausschließlich aufgrund ökonomischer Bestimmungsfaktoren (Preise) determiniert wird.

\section{Der energietechnisch orientierte Ansatz (Mikroanalyse)}

Dieser geht aus von den einzelnen Bereitstellungstechniken für die verschiedenen Energieverwendungszwecke in den energienachfragenden Sektoren $[5,6]$. Ännlich wie im makroökonomischen Ansatz wird auch hier versucht, den Energiebedarf nicht isoliert zu beschreiben, sondern, wie aus Bild 1 ersichtlich, ihn einzubetten in den Gesamtzusammenhang zwischen Wirtschaft, Energieversorgung und Umwelt. Diese Vorgehensweise ermöglicht nicht nur eine Erfassung der wesentlichen Determinanten des Energiebedarfs, sondern sie stellt auch sicher, daß die sich aus den Letztverwendungszwecken der Energie in Verbindung mit den eingesetzten Bereitstellungstechniken ergebenden Anforderungen, z.B. hinsichtlich notwendiger Leistungen und Reservehaltung, berücksichtigt werden.

In Bild 2 ist schematisch der Aufbau eines an den verschiedenen Bereitstellungstechniken orientierten Energiebedarfmodells dargestellt. Es verknüpft zunächst die jeweilige Bereitstellungstechnik mit dem letztlich beabsichtigten Zweck des Energieeinsatzes. In der Abb. ist beispielhaft die Befriedigung des Bedürfnisses nach angemessenem Wohnraum und behaglichem Wohnklima herausgestellt. Unter Berücksichtigung der den Wärmebedarf bestimmenden Gebäudeparameter wird der notwendige Nutzenergiebedarf ermittelt. Hierunter ist im Beispiel die von dem Raumheizsystem abzugebende Wärme zu verstehen. Hieran anschließend können die Möglichkeiten, Voraussetzungen und Auswirkungen prozeßtechnisch alternativer Deckung des jeweiligen Nutzenergiebedarfs untersucht werden. Ausgehend von der 


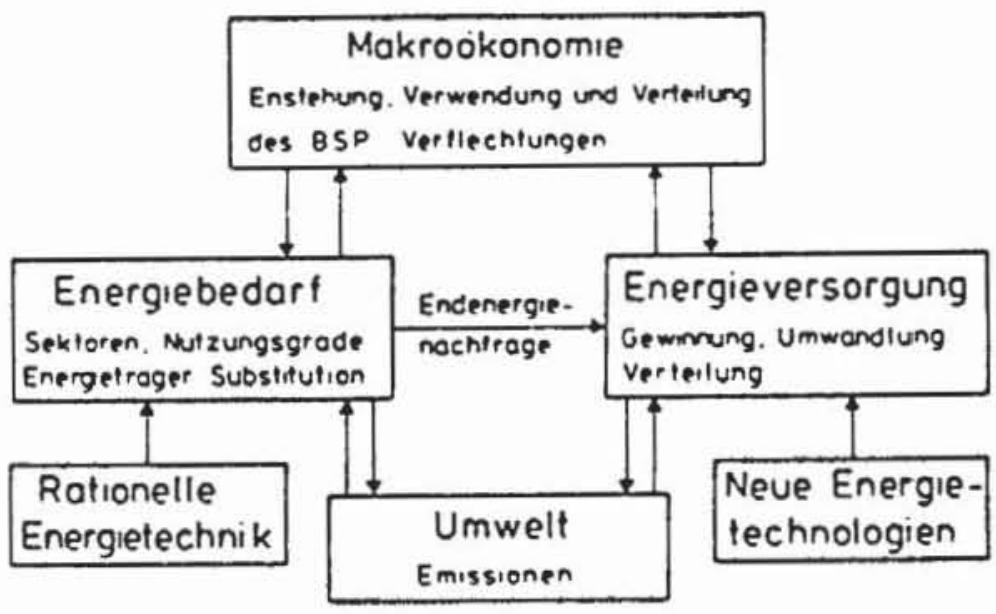

Bild 1: Wirtschaft, Energie und Umwelt

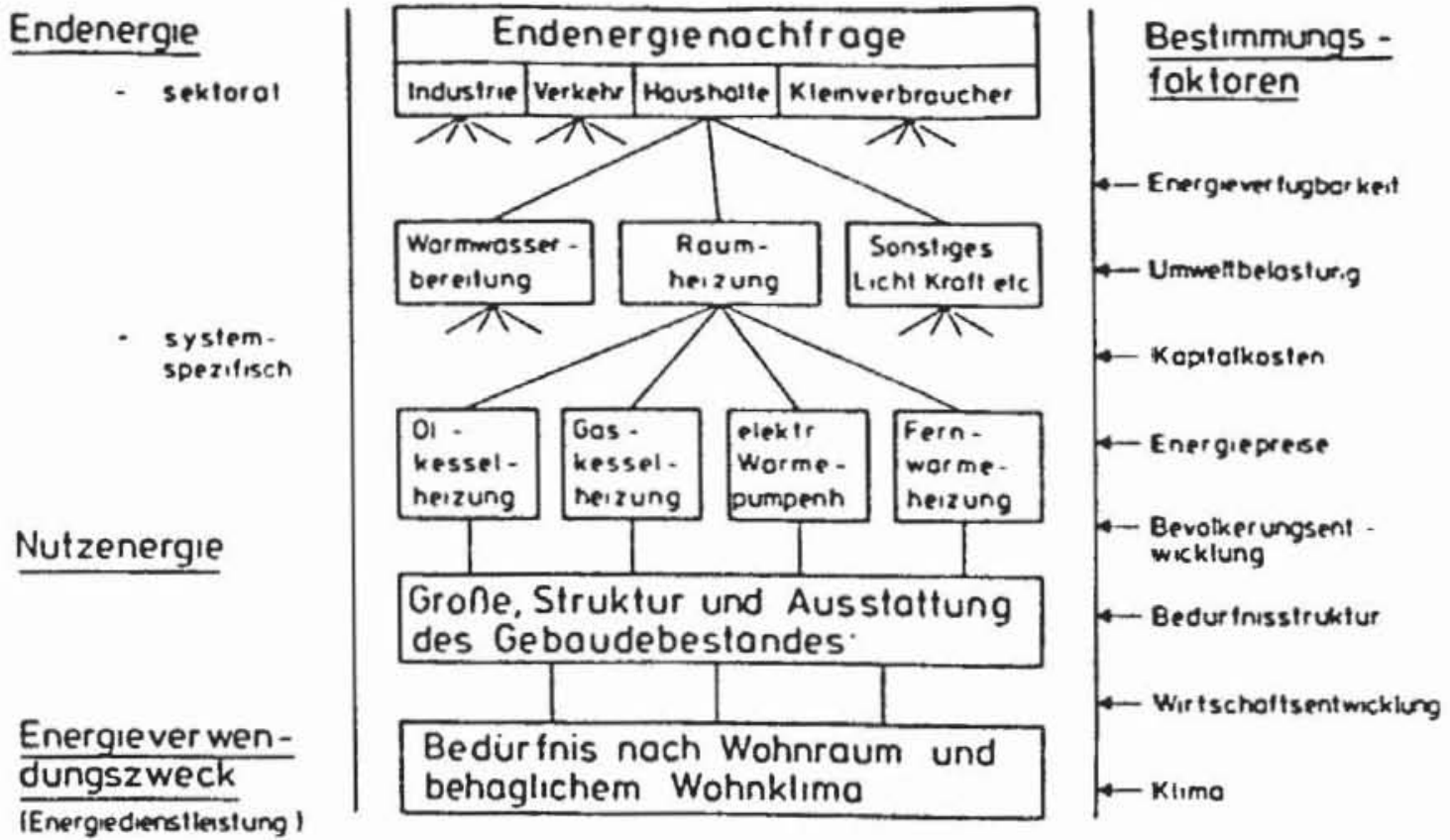

Bild 2: An den Bereitstellungstechniken orientiertes Energiebedarfsmodell

Höhe und der Leistungscharakteristik des Nutzenergiebedarfs wird über die Anteile der sektoral zum Einsatz kommenden Endbenutzertechniken, im skizzierten Fall über die verschiedenen Heizungssysteme, der für den jeweiligen Verwendungszweck erforderliche sektorale Endenergiebedarf bestimmt. Integriert über alle energieeinsetzenden Sektoren ergibt sich dann der gesamte Endenergiebedarf, der vom Energieversorgungssystem bereitgestellt werden muß. Eine derartige Vorgehens- 
weise ermöglicht eine realistische, an den technischen Möglichkeiten orientierte Beschreibung des Endenergiebedarfs unter Berücksichtigung der Einspar- und Substitutionsmöglichkeiten.

Der Vorteil des energietechnisch-orientierten Ansatzes gegenüber dem makroökonomischen ist neben dem größeren Detaillierungsgrad darin zu sehen, daß er der formalen Beschreibung einer Substitution von Energie durch Kapital einen materiell technischen Inhalt verschafft und auch Antworten geben kann auf die Frage, wo diese Substitution ihre naturgesetzlich technischen und ihre von den Nutzerkriterien beeinflußten Grenzen hat.

Natürlich stehen der Realisierung einer derart detaillierten Beschreibung der Transformation von Bedürfnissen in den Bedarf an Endenergie etliche noch ungelöste Probleme entgegen. Schwierig gestaltet sich insbesondere die Verknüpfung des Nutzenergiebedarfs mit den jeweils bedarfsbestimmenden Faktoren. Die Schwierigkeiten sind zum einen in der Vielzahl der das Konsumentenverhalten bestimmenden Faktoren zu sehen. Zum anderen sind in vielen Fällen die funktionalen Zusammenhänge zwischen Bedürfnissen und ihren Bestimmungsfaktoren gar nicht oder nur qualitativ bekannt, was insbesondere zu enormen Unsicherheiten bei der Quantifizierung der zukünftigen Bedürfnisentwicklung führt. Dies gilt nicht nur für die dem gesellschaftlichen und sozialen Bereich zuzuordnenden Einflußgrößen, sondern auch für viele technisch-ökonomische Faktoren. So z.B. sind heute Informationen über die Kosten und den Nutzen von energiesparenden Maßnahmen in vielen Bereichen überhaupt nicht oder nur bruchstückhaft verfügbar. Hier bietet sich noch ein breites Betätigungsfeld für die Energiebedarfsforschung. Dies gilt auch für die Untersuchung der praktisch äußerst wichtigen, prozeßtechnischen Zusammenhänge zwischen Energieeinsatz oder besser Exergieverbrauch und zeitlichem Aufwand. Wir benutzen heute in vielen Fällen Energie, um Zeit zu sparen. Schnellere Verkehrssysteme sind dafür ein direkt einsichtiges Besipiel. Aber auch in vielen Produktionsprozessen nehmen wir Energieverluste in Kauf, um Zeit zu sparen. Quantitativ sind diese Zusammenhänge aber noch wenig erforscht.

\section{Zusammenfassung und Ausblick}

Wenn strategische Energiemodelle einen realistischen Beitrag zur Entscheidungshilfe liefern sollen, d.h. wenn es gilt, Handlungsoptionen auf dem Energiegebiet unter Beachtung deutlich erkannter $Z$ wangsläufigkeiten, weniger überschaubarer $\mathrm{Zu}$ sammenhänge und unsicherer Zukunftserwartungen auf ihre Voraussetzungen und Auswirkungen hin zu untersuchen und zu bewerten, so müssen die Modelle den Eventualitäten und Modalitäten des künftigen Energiebedarfs Rechnung tragen. Die Praxis der Energiemodelle geht von zwei unterschiedlichen Ansätzen aus: Während der makroökonomische Ansatz, nach dem Vorbild der ökonomischen Modelle ange- 
legt, Energie undifferenziert als einen Produktionsfaktor der sektoralen oder gesamtwirtschaftlichen Produktionsfunktion auffaßt, erfaßt der energietechnisch orientierte Ansatz den spezifischen Energiebedarf in Verbindung mit dem jeweils beabsichtigten Energieverwendungszweck. Er wird daher den Modalitäten des Energieeinsatzes in stärkerem Maße gerecht. Die Konzeption des Energieverbrauchs als einer notwendigen Begleiterscheinung volkswirtschaftlicher Aktivität, auch wenn sie sektorspezifisch erfolgt, ist viel zu generell, um den Möglichkeiten der Energietechnik und der Anderung im Verbraucherverhalten allein schon über sektorale Korrelationen gerecht zu werden.

Beide Ansätze sehen sich der Schwierigkeit konfrontiert, die bedarfsbestimmenden Faktoren und ihre künftige Entwicklung und thre Wirkung auf den Energieeinsatz adäquat abzubılden. Das erfordert, auf eıner stark empirie-orientierten Grundlage, die Ableitung quantitativer bzw. formaler Zusammenhänge zwischen Energienachfrage und bedarfsbestimmenden Größen, wobei moglichst die jeweiligen Energieverwendungszwecke und Prozeßschritte konkretisiert werden sollten. Das erfordert ebenfalls die Verdichtung einer Vielfalt von wirksamen Einflußfaktoren zu strategisch gewichtigen Kenngrößen. In beiderlei Arbeitsrıchtung bleibt noch vieles zu tun. Die zweifelsohne heute noch bestehenden Wissens- und Datenlücken sollten aber nicht zu der Schlußfolgerung führen, daß mit dem vorhandenen Wissen und den verfügbaren Daten und methodischen Ansätzen nicht schon heute brauchbare Aussagen in bezug auf den Energiebedarf und die sich daraus ergebenden Anforderungen an den Ausbau der Energieversorgung möglich sind.

\section{$\underline{\text { Literaturangaben }}$}

[1] A. Voß

Ein Energiemodellsystem zur Analyse der Entwicklungsmöglichkeiten der Energieversorgung, in Großtechnische Energienutzung und menschlicher Lebensraum, IIASA, Wien 1977

[2] A. Voß

Möglichkeiten und Grenzen von Energieprognosen, in Existenzfrage: Energie, Econ-Verlag, Düsseldorf 1980

\section{[3] A. Berckmans, et al}

The macroeconomic energy model EURECA, in Prodeedings of the International Conference on Energy Systems Analysis;

D. Reidel Publishing Company, Dordrecht, Holland 1980 
[4] J. Seetzen, et al

Makroökonomische Input-Output-Analysen und dynamische Modelle zur Erfassung technischer Entwicklungen, ISR 69, Birkhäuser Verlag, Basel 1979

[S] H. Reents

Die Entwicklung des sektoralen End- und Nutzenergiebedarfs in der Bundesrepublik Deutschland, Bericht der KFA Jülich, Jül-1452, Jülich 1977

[6] A. Voß, K. Schmitz (Hrsg.)

Energiemodelle für die Bundesrepublik Deutschland, Verlag TÜV Rheinland $\mathrm{GmbH}$, Köln 1980

[7] H. Matthöfer (Hrsg.)

Energiebedarf und Energiebedarfsforschung, Argumente in der Energiediskussion, Bd. 2, Neckar Verlag, Villingen 1977 